\title{
Auxanographic Techniques in Biochemical Genetics
}

\author{
By G. PONTECORVO \\ Department of Genetics, The University, Glasgow
}

SUMMARY : Details are given of the 'auxanographic' method for identifying growthfactor requirements of micro-organisms, and particularly of mutant strains differing from a parent strain in having additional growth-factor requirements. Cells are distributed in a nutritionally deficient agar medium which is allowed to set in a Petri dish. Possible required nutrients are then spotted on the agar surface, thus enabling the corresponding exacting mutants to grow at those localities. The technique is only a labour-saving device which can be extremely efficient in shortening the preliminary steps in the work. It can also be applied to other uses, such as the identification of competitive interactions between nutrilites.

Sixty years ago, in 1889, Beyerinck (Beijerinck) described a technique, simple and efficient, for the study of the nutritional requirements of micro-organisms. The 'auxanographic' technique, as he called it, has gone almost unnoticed; the present author became aware of Beyerinck's paper only after having redevised the technique, developed it for biochemical genetics, and used it for several years (Pontecorvo, 1947). Its efficiency in saving labour and its many uses make worthwhile a description of its applications to the study of the genetics of micro-organisms and certain other problems.

On a densely seeded agar plate of a medium lacking a required growth factor for a given micro-organism, no visible growth can take place. If, however, we place at a point of the agar surface a minute amount of the missing growth factor, this will diffuse out, and on incubation growth will take place in a circular area centred on that point. Instead of a growth response other kinds of visible response can be used; Beyerinck suggested, for instance, luminescence, secretion of a pigment, etc.

The technique can be used in reverse, as, indeed, it has been used in Heatley's cylinder assay for penicillin and its numerous variations. If we place a minute amount of an inhibitory substance at a point on the agar surface of a seeded nutrient medium, a circular clear area without growth, surrounded by a general background with growth, will remain after incubation.

The first kind of test, based on the promotion of growth, could perhaps be termed 'positive' auxanography; the second, based on inhibition of growth, 'negative' auxanography. The two are sometimes used in combination, for instance, for investigating the mode of action of a metabolite analogue (see below), and are suitable for quantitative as well as qualitative tests. An example of quantitative positive auxanography is the plate assay for growth factors (see below), and an example of quantitative negative auxanography is the cylinder assay for penicillin. Qualitative negative auxanography has been used by a number of authors especially for detecting the presence of antibiotics in culture filtrates and their spectrum of activity. Qualitative positive auxanography has been extensively used by the present author for a quick 
characterization of the nutritional requirements of mutant strains as compared with those of a parent strain; the present paper describes mainly the technique of qualitative positive auxanography as used for this purpose.

\section{Qualitative positive auxanography for the screening of nutritional mutants}

With Beadle's technique, devised originally for Neurospora, but subsequently adapted to other moulds, bacteria and yeasts, nutritionally exacting mutant strains are identified as such in so far as they cannot grow on the simplest chemically defined medium ('minimal') sufficient for growth of the parent strain, but can grow on a complex medium ('complete') containing a large variety of known and unknown organic compounds. After identification, isolation, and genetical or other tests, a mutant has to be characterized, i.e. a search is made for the simplest growth factor, or combination of growth factors, which will make growth possible when added to the minimal medium. In other words, there must be identified the growth factor, or combination of growth factors, present in the complete medium but not in the minimal medium, which account for the growth of the mutant on the former and not on the latter. It is in this characterization of the growth requirements of a mutant, particularly in the earlier stages, that the auxanographic tests have proved to be invaluable.

\section{EXPERIMENTAL}

The technique used in the case of Aspergillus nidulans will be given here; only minor variations are required for use with other micro-organisms. A. nidulans is a filamentous fungus, most naturally occurring strains of which grow on a minimal medium made up as follows: $25 \mathrm{~g}$. glucose; $15 \mathrm{~g} . \mathrm{NaNO}_{3} ; 1 \cdot 3 \mathrm{~g}$. $\mathrm{MgSO}_{4} \cdot 7 \mathrm{H}_{2} \mathrm{O} ; \mathbf{1 . 3} \mathrm{g} . \mathrm{KCl} ; 3 \cdot 8 \mathrm{~g} . \mathrm{KH}_{2} \mathrm{PO}_{4} ; 10 \cdot 0 \mathrm{mg}$. $\mathrm{FeSO}_{4} \cdot 7 \mathrm{H}_{2} \mathrm{O} ; 1.0 \mathrm{mg}$. $\mathrm{ZnSO}_{4} \cdot 7 \mathrm{H}_{2} \mathrm{O}$; distilled water $2500 \mathrm{ml}$. This medium is solidified with $1.5 \%$ agar. The agar is purified by washing in running water for at least $2 \mathrm{hr}$.

The normal strain will, of course, grow equally well or better on complex media supplying many additional substances. One of these 'complete' media is the following: glucose, $10 \mathrm{~g}$.; peptone, $10 \mathrm{~g}$.; 'Yeastrel', $5 \mathrm{~g}$.; tap water $1000 \mathrm{ml}$. Any brand of peptone or of yeast extract can be used.

With each mutant strain, obtained by one of the various techniques available, a search is made for the substance (or substances) present in the complete medium, which is necessary as a supplement to the minimal medium to make possible the growth of the mutant on it.

Petri dishes of minimal medium agar are inoculated by adding to the cooled $\left(50^{\circ}\right)$ molten agar a heavy inoculum of the strain to be tested. This inoculum should secure potential uniform growth in the whole plate; i.e. at least, $1,000,000$ conidia/Petri dish. The inoculated plate is incubated for $6 \mathrm{hr}$. or more to allow the spores to germinate, and after that, minute amounts of various substances are placed in marked positions on the agar surface; a straight platinum wire with which each substance has been just touched provides enough material. When one of these substances, or mixtures of substances, 
is capable of promoting the growth of the mutant, after further incubation for $24 \mathrm{hr}$. a circular area of growth appears around the position where the successful substance has been put. For most purposes the substances to be tested need not be sterile; the high concentration at the point of inoculum usually takes care of that.

The auxanographic test is carried out in successive steps by using arrays of substances which progressively restrict the field of search. In routine work for the characterization of mutants it has been found useful to proceed as follows. In the first step three materials: vitamin-free casein hydrolysate (Ashe Laboratories Ltd.), supplemented with tryptophan; yeast nucleic acid (British Drug Houses Ltd.); and 'Yeastrel', are tested simultaneously on the same Petri dish. The yeast extract is used in even smaller amounts than the other two ingredients (see above). Two or three Petri dishes are usually enough to give an indication of consistent, as distinct from accidental, response.

According to the results obtained from the first step, the second step will be in one or other direction. For instance, response to casein acid-hydrolysate, but not to yeast nucleic acid, and a very small response to yeast extract, will suggest the use of various amino-acids in the second step. On the other hand, good response to yeast extract, but not to yeast nucleic acid nor to casein hydrolysate points to a need for vitamins of the B group.

It is cheaper and almost as efficient to use in the first step the materials mentioned rather than to follow the more precise and reliable method of using only mixtures of known chemical compounds, and omitting one or more components in successive tests. After the first step, the successive ones are, of course, carried out with known mixtures of chemically defined substances or with single substances. Once a particular substance has been identified as capable of promoting growth of a mutant, the further tests for establishing whether this is the only one capable of doing so have to be carried out with the usual biochemical procedures, though the auxanographic test can go a long way towards the identification of the simplest effective substance.

In view of the particular interest which, at the present moment, centres on the biosynthesis of polypeptides, the use in the first auxanographic test of enzymic partial digests of vitamin-free casein at the same time as casein acidhydrolysate, may be rewarding. Response to the former, but not to the latter, would indicate a requirement for a polypeptide.

An example of the characterization of a mutant of Aspergillus nidulans is given in Pl. 1, figs. 1-2. The mutant turned out to be one requiring for growth the addition of lysine to the minimal medium. Fig. 1 shows the response to the three materials mentioned before. It is clear that casein acid-hydrolysate is effective, while yeast nucleic acid and yeast extract are not. Fig. 2 shows the subsequent test, i.e. that of a number of amino-acids suggested by the results of the first test; there is a clear response to one of them (lysine) but not to any of the others. A different mutant gave good response to both casein acidhydrolysate and yeast extract. A response of this kind suggests the effectiveness of both a vitamin of the B group and an amino-acid. Among other compounds, nicotinic acid and tryptophan were tested in the second-stage auxanographic 
test and were both found to be effective. In a third test some of the presumed intermediates and precursors of tryptophan and nicotinic acid were tested, and the mutant was found to respond to anthranilic acid, indole, kynurenine and nicotinic acid (Pl. 1, fig. 3).

The auxanographic test can reveal also cases of double requirements (i.e. two substances simultaneously required for growth) and cases of inhibition of the biological activity of one substance by another. An example of the first kind is given in Pl. 1, figs. 4, 5, which show the simultaneous effect of aneurin and thiosulphate on a mutant of $A$. nidulans which requires both because of two independent mutational changes. The test can be done either, as fig. 4 shows, by using cylinders containing the two solutions, or by placing the crystals of the two substances directly on the agar surface. In the latter case, a further refinement is that of streaking the two substances at right angles; the resulting area of growth will be determined by the co-ordinates of the concentrations of both substances which are compatible with growth (Pl. 1, fig. 5).

Another way in which the auxanographic test can be used is in the identification of the inhibitory action of one substance on the biological activity of another one. Fig. 6 (Pl. 1) shows a test in which the mentioned lysine-requiring mutant of $A$. nidulans was tested for possible inhibition by arginine. Arginine and lysine were streaked on the plate at right angles as uniformly as possible along the length of the streaks. The resulting shape of the area in which growth took place indicated the mode of inhibition of lysine by arginine. It is clear that over a large range of concentrations, the inhibitory level of arginine was independent of the concentration of arginine, but dependent on the ratio of the concentration of arginine to that of lysine. In other words, the approximately straight line which limited the area of growth from that of inhibition indicated that the type of interaction between the two substances was probably a competitive one. It is clear that in many cases competitive interaction may be discovered by this simple procedure, which requires only one or a few Petri dishes and overnight incubation.

\section{Plate assays for growth factors}

The use of positive quantitative auxanography for the assay of growth factors has been only recently developed in industrial laboratories on a practical scale, though it had been implicit in the auxanographic tests described by the author in the last few years (Pontecorvo, 1947). Fig. 7(Pl. 1) shows a plate assay carried out with a mutant of $\boldsymbol{A}$. nidulans which requires biotin. In this case the method used was that of soaking standard small disks of sterile filter-paper in solutions containing various concentrations of biotin and placing them on to the surface of the agar. Cylinders, instead of disks of filter-paper, can obviously be used, as well as any other of the various devices developed for plate assays of antibiotics. The great advantage of positive auxanography as a method of microbiological assay lies in the possibility of using moulds, and especially mutants of moulds, which are at present being produced in large numbers in laboratories all over the world, without having to weigh the mycelium to estimate the amount of growth. 


\section{CONCLUSION}

The auxanographic method, adapted to various needs and various organisms, can be of invaluable help in many directions, but especially in the characterization of the nutritional requirements of micro-organisms. It is, obviously, only a relatively crude method, and its aim is only that of decreasing the amount of labour involved in restricting the field of search for exact nutritional requirements. It is probably adaptable to the identification of co-factors required by lysins to lyse cells, by bacteriophages to become adsorbed on to the host, and by bacterial or other enzymes to give colour, or other visible, reactions.

I am indebted to Mr E. Forbes for invaluable technical assistance. Part of the work was supported by a grant for special research from the Department of Scientific and Industrial Research.

\section{REFERENCES}

Beyerinck, M. W. (1889). L'auxanographie, ou la méthode de l'hydrodiffusion dans la gélatine appliquée aux recherches microbiologiques. Arch. néerl. Sci. 23, 367.

Ponteconvo, G. (1947). The genetical control of nutritional requirements in microorganisms and its application to microbiological assays. Proc. Nutrit. Soc. 5, 182.

\section{EXPLANATION OF PLATE}

Fig. 1. First step in the auxanographic test; effect of casein acid-hydrolysate (plus tryptophan), yeast nucleic acid and yeast extract. The figure shows a mutant of Aspergillus nidulans responding to casein hydrolysate.

Fig. 2. Second step. The same mutant, tested for response to various amino-acids; response only to lysine.

Fig. 3. A mutant of $A$. nidulans which responded to both casein acid-hydrolysate and yeast extract, is tested for response to possible intermediates in the synthesis of nicotinic acid.

Fig. 4. A 'double' mutant of $\boldsymbol{A}$. nidulans requiring both aneurin and thiosulphate. The two substances diffuse out from the cups and growth takes place where the concentration of both is above a certain level.

Fig. 5. Same test as fig. 4, but each substance is laid along one of the two dark lines.

Fig. 6. The lysine-requiring mutant, of figs. 1 and 2 , is tested for a possible competitive inhibition by arginine. The lysine was laid along a streak at right angles to the arginine streak.

Fig. 7. Plate assay of biotin with a biotin-requiring mutant of $\boldsymbol{A}$. nidulans; concentrations of biotin in $\mu \mathrm{g} \cdot / \mathrm{ml}$.

(Received 31 May 1948)

Addendum (18 November 1948). I am indebted to Professor Gäumann for calling my attention to two papers reporting extensive use of the auxanographic technique.

GäUMANN, E. (1921). Over een bakterielle vaatbundelziekte der bananen in Nederlandsch-Indie. Mededeelingen van het Instituut voor Plantenziekten, No. 48. Batavia.

HaLl, C. J. J. van (1902). Beijdragen tot de kennis der bakterielle plantenziekten. Thesis, Amsterdam University. 
Journal of General Microbiology, Vol. 3, No. 1

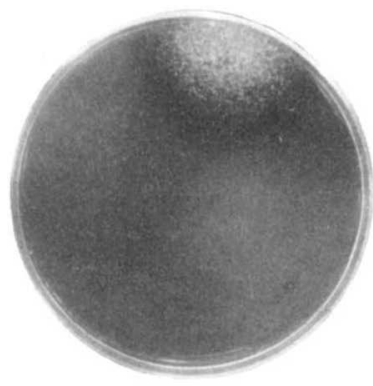

l'ig. 1

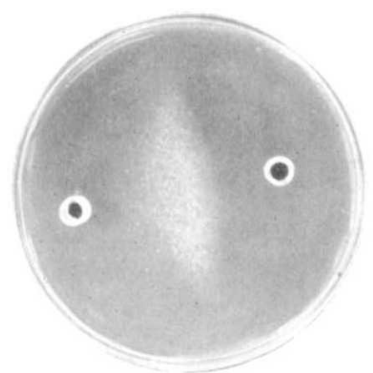

rig. +

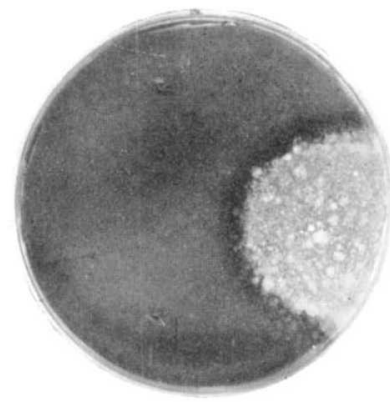

Fig. 2

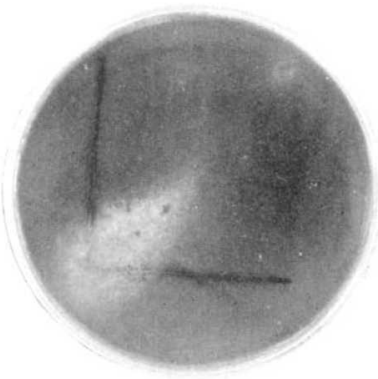

Fig. 5

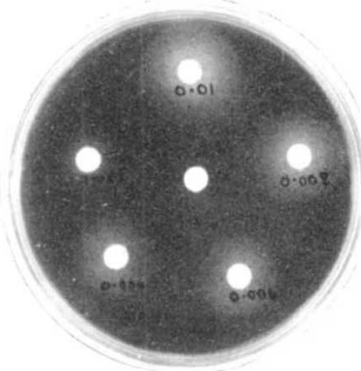

Fig. 7

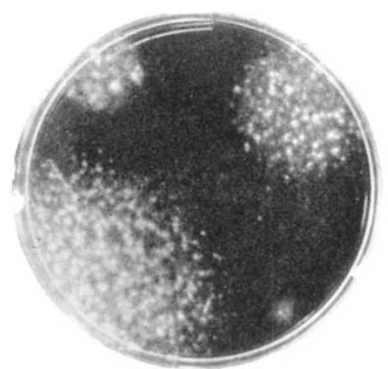

Fig. 3

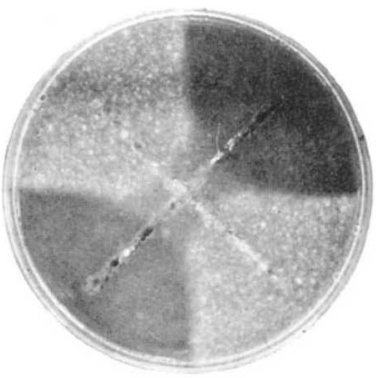

Fig. o

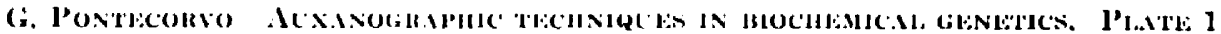

